\title{
Beiträge zur Entwickelungsmechanik der aus einem Ei entstehenden Doppelbildungen.
}

\author{
Von \\ Jacques Loeb, \\ University of Chicago.
}

Mit Tafel XVIII-XIX und 20 Figuren im Text.

Eingegangen am 3. Januar 1895.

I.

Über die Umstände, welche die Entstehung von Doppelbildungen bestimmen, hatte man bis auf die jünste Zeit nur unvollkommene Vorstellungen. Die Versuche Trembley's, Rösel's, Bonnet's, SpalLANZANI's uber Regeneration mussten jeden an causales Denken gewöhnten Biologen zum Schluss führen, dass auch Zwillingsbildungen in einem Ei nur dadurch zu Stande kommen, dass der Inhalt des Eies gespalten wird. Schneidet man eine Hydra oder Planarie in $\mathbf{z w e i}$ Stiicke, so erhält man statt des einen Individuums deren zwei. So sehen wir auch in der That, dass die älteren Physiologen, die sich tuber Zwillingsbildung aussprechen, logischer Weise die Entstehung von mehr als žwei Individuen aus einem Ei auf eine Spaltung des Keimes zurückführten. Am klarsten erscheint das bei Jон. MülLLER: „Die Theile sind auf dieser niedersten Stufe der Entwickelung noch so wenig verschieden und enthalten so gleich viel vom Ganzen, dass sie selbst das in ihnen vom Ganzen enthaltene zum Ganzen ausbilden können. Diese Art der Doppelbildung durch Theilung muss daher den niedersten Thierformen zukommen. Aus gleichen Grunden muss diese Art der Doppelbildung dem Embryo des Menschen und der Thiere zu einer Zeit zukommen können, wo das Einzelne noch im Ganzen und vom Ganzen gleichviel in den Archiv f. Entwickelungsmechanik. I. 
verschiedenen Theilen enthalten ist. Wenn nun äußere oder innere Ursachen dieser theilweisen Spaltung des noch unentwickelten Grundstoffes vorhanden sind, so müssen beide Theile das in ihnen vom Ganzen enthaltene zur Doppelbildung ausscheiden ${ }^{1) . *}$ Wenn man von der naturphilosophischen Ausdrucksweise absieht, könnte dieser Ausspruch als ein präcises Résumé alles Dessen gelten, was in letzter Zeit uber den Gegenstand experimentell festgestellt wurde. Es blieb indessen nicht bei bloßen Vermuthungen. Valentin prüfte diese Ideen experimentell und es gelang ihm durch künstliche Spaltung des Htihnerkeimes Doppelbildungen hervorzubringen. Bei einem 2 tägigen Embryo gelang es ihm beispielsweise durch Spaltung des hinteren Endes Verdoppelung des Beckens und der hinteren Extremitäten herbeizuführen ${ }^{2}$ ).

Man hätte erwarten sollen, dass die Wissenschaft nunmehr die sich ans den Regenerationserscheinungen fur das $\mathrm{Ei}$ ergebenden Probleme mit Rücksicht auf deren fundamentale Bedeutung in gerader Linie weiter verfolgen würde. Das war nicht der Fall. Die fruchtbare Anwendung der Physik auf die Physiologie in den vierziger Jahren lenkte die Aufmerksamkeit der Physiologen von diesem Gebiete ab, und den Morphologen lag das causale Denken und Arbeiten tiberhanpt weniger nahe. Im Anschluss an die Arbeiten DARwIN's wurde die deskriptive Entwickelungsgeschichte allerdings mächtig gefördert, allein die wirklich fruchtbare Seite der darwinistischen Ideen, nämlich die experimentelle, wurde so gut wie ganz übersehen. Statt dessen aber schoss das Unkraut romanhafter Spekulationen mächtig auf.

Unabhängig von dieser romanhaften Strömung, theilweise sogar derselben direkt entgegengerichtet, entstand in den letzten Jahrzehnten auf botanischer Seite die causale Morphologie von SACHS, deren Principien in den beiden klassischen Abhandlungen über "Stoff und Form der Pflanzenorgane «3) (1880) zusammengefasst wurden; anf zoologischer Seite leiteten die experimentellen Arbeiten der Gebriider Hertwig, His, Pflüger und Roux u. A. auch wieder

1) J. MÜller, Über die Metamorphose des Nervensystems, Meckel's Archiv 1828. (Ich citire nach WIEDEMans, Ưber die Entstehung ron Doppelbildungen. VrRchow's Archiv 138, pag. 178.)

2) WAGNeR's Handwörterbuch der Physiologie, Bd. I. pag. 913. (VALENTix's Versuche wurden 1837 veröffentlicht.)

3) Julius Sachs, Gesammelte Abhandlangen über Pflanzen-Physiologie. Bd. II. 1893. 
Beitr. z. Entwicklgsmech. d. aus einem Ei entstehend. Doppelbildungen. 455

zu den Problemen, die schon Joh. Müller und Valentin beschäftigt hatten.

Über die Mechanik der Doppelbildungen gewährten die Arbeiten von Rovx die ersten weiteren Aufschlïsse. Rocx fand, dass nach Tödtung einer der beiden Zellen des ersten Furchungsstadiums eines Froscheies die am Leben gebliebene sich zunächst zu einem halben Embryo entwickelt, dass aber durch einen Regenerationsprocess ein ganzer Embryo aus der einen Furchungskugel entstehen könne. Er weist auf die Möglichkeit hin, dass bei ausbleibender Vereinigung der Medullarwülste durch regenerative Processe jede Hälfte zu einem vollständigen Embryo werden könne und dass auf diese Weise Doppelbildungen in einem Ei entstehen können ${ }^{1}$ ). Diese Regenerationsprocesse sind von 0 . Невтwig in $\mathrm{Zweifel}$ gezogen, allein die für uns wesentliche Thatsache, dass aus einer der beiden ersten Furchungskugeln des Froscheies ein ganzer Embryo entstehen könne, ist auch von Hertwig festgestellt worden ${ }^{2}$ ). Chabry hat bei Ascidieneiern eine Furchungskugel des Zweizellenstadiums zerstört und ebenfalls konstatirt, dass eine Zelle allein sich zu einem ganzen Embryo entwickeln könne ${ }^{3}$ ). Driesch zerstörte nach HertwiG's Methode die Membran bei Seeigeleiern, indem er dieselbe im ersten oder späteren Furchungsstadien schüttelte ${ }^{4}$. Befinden sich solche Eier im Zweizellenstadium, so kann leicht eine einzelne Zelle aus dem Ei geschleudert werden. In dem Falle entwickelte sich die zurïckgebliebene Zelle za einem vollständigen Embryo. Es müssen also, wenn beide Zellen getrennt werden, Zwillinge entstehen. War die Eihaut durch das Schïtteln nicht gesprengt, so kam es relativ häufig vor (in 6 unter 41 Fällen), dass Zwillinge im Ei sich bildeten. Nach Driesch war in diésen Fällen der Kontakt der Furchungszellen ziemlich gelockert. - VeJDovskí hatte bei Allolobophora Zwillingsbildungen beobachtet und vermuthet, dass sie eine Folge höherer Temperatur seien. DRIEsch 'priifte diese Vermuthung bei Seeigeleiern und fand, dass in der That, wenn dieselben einer

1) W. Roux, Beiträge zur Entwickelungsmechanik des Embryo. Vxrchow's Archiv, Bd. 114. 1888.

2) 0. Hertwig, Über den Werth der ersten Furchungszellen für die Organbildung des Embryo. Arch. f. mikrosk. Anat., 42. Bd. 1893.

3) Contribution à l'embryologie normale etc. Journal de l'anat. et de la phys. 1887 (war mir nicht zugänglich).

4) H. DrIEsch, Entwickelungsmechanische Studien. I. II. Zeitschrift für wissensch. Zoologie. Bd. 53. 
Temperatur von ca. $31^{\circ}$ hinreichend lange ausgesetzt blieben, während die Temperatur des Seewassers vorher $14-18^{\circ}$ betragen hatte, nicht selten Zwillingsbildungen resultiren ${ }^{1}$ ). Die ersten Furchungskugeln sahen dann so aus, »als wären sie aus einander gerissen worden, sie berühren sich nicht». Diese Versuche beweisen deutlich, dass Trennung der Furchungskugeln zu Zwillingsbildungen führt.

Ist nun damit die Richtigkeit der Spaltungstheorie von JoH. MüLLER nachgewiesen, so bleibt es doch noch fraglich, welche Kräfte im Uterus eine Spaltung des Keimes herbeiftuhren können. Trennung derFurchungskugeln durch Schutteln ist hier ausgeschlossen, ebenso, bei derKleinheit desEies, Trennung derselben durch Kompression des Eies. Von einer erhöhten Temperatur des Uterus kann nicht die Rede sein, jedenfalls nicht von einer solchen Temperaturerhöhung, die nach DrIEsch's Versuchen zu Zwillingsbildungen nöthig ist, die ja nicht einmal im Fieber erreichbar wäre. Ich fand nun später eine Methode, Doppelbildungen durch solche Kräfte künstlich herbeizuführen, die im Uterus wenigstens zur Geltung kommen könnten, nämlich osmotische Kräfte. Wenn ich befruchtete Seeigeleier in verdünntes Seewasser brachte, so nahm das Ei in Folge der osmotischen Druckdifferenz zwischen Zellsaft und Seewasser Wasser anf und die dadurch bedingte Volumzunahme führte zum Platzen der Membran. Ein Theil des Protoplasmas floss aus, nahm Kugelform an und das Ei wurde so in eine Doppelkngel umgewandelt. Jede der beiden Kugeln kann sich zu einem ganzen Embryo entwickeln 2). - Im letzten Jahre hat weiter O. Schultze die interessante Entdeckung gemacht, dass aus Froscheiern, die nach der ersten Furchung um $180^{\circ}$ gedreht werden, sich relativ hänfig Doppelembryonen entwickeln 3 ).

Die Hauptaufgabe, die noch zu erledigen ist, besteht in einer Theorie der Vorgänge, die zu einer Spaltung des Keimes führen. Wie kann eine Temperaturerhöhung oder eine Anderung der geotropischen Orientirung des Eies etc. eine Trennung der beiden ersten Furchungszellen zur Folge haben? Der zweite Theil der folgenden Arbeit soll einen Beitrag zur Beantwortung dieser Frage liefern.

1) DrIEsch, Entwickelangsmechanische Studien. III.-VI. Zeitschrift für wissensch. Zoologie. Bd. 55, 1892.

2) LoEb, Über eine einfache Methode zwei oder mehr zasammengewachsene Embryonen ans einem Ei hervorzubringen. Pflüger's Archiv, Bd. 55. - Biological Lectures delivered at Words Holl, GrNN \& Co. Boston 1893. - Über die Grenzen der Theilbarkeit der Eisubstanz. PfLüGer's Archiv Bd. 59.

3) 0. Schultze, Verhandl. der anat. Gesellsch. 1894. pag. 117. 
Beitr. z. Entwicklgsmech. d. ans einem Ei entstehend. Doppelbildungen. 457

Im ersten Theil will ich die Entstehung von Doppelbildungen aus dem künstlich in eine Doppelkugel umgeformten $\mathrm{Ei}$ vom entwickelungsmechanischen Standpunkt behandeln. Dabei will ich es möglichst vermeiden auf die Einzelheiten zurückzukommen, die ich schon früher über den Gegenstand mitgetheilt habe.

\section{Ober Furchung und Blastulabildung der in eine Doppelkugel umgeformten Eier.}

1. Entwickelungsmechanisch lässt sich die Frage aufwerfen, was aus einem befruchteten ungefurchten $\mathrm{Ei}$ wird, wenn man folgende zwei Veränderungen mit ihm vornimmt. 1) Die geometrische Form des Eies werde derartig verändert, dass statt der gewöhnlichen Kugelgestalt (Textfig. 1) das Ei die Form einer Doppelkugel (Textfig. 2) annimmt. 2) Die Beschränkung der Bewegung der einzelnen Furchungszellen durch die Membran werde entweder ganz auf-

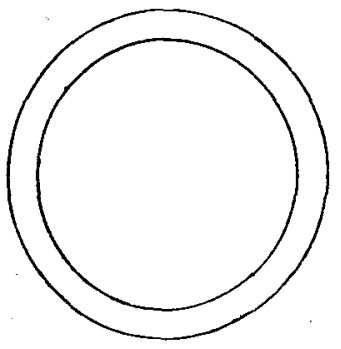

Fig. 1.

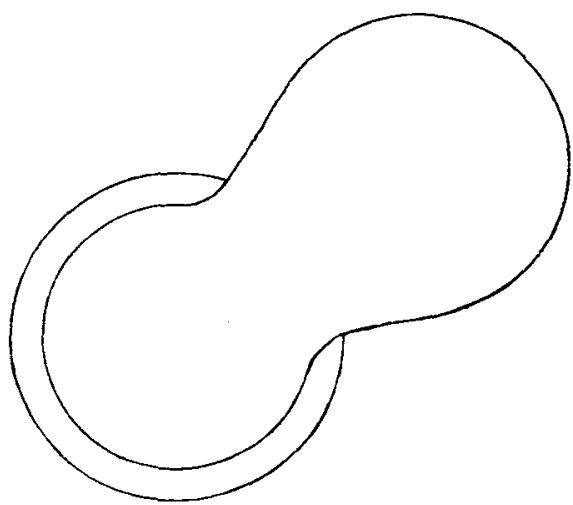

Fig. 2 .

gehoben oder bleibe nur bei einer der beiden Kugeln erhalten. Die Methode, deren ich mich bediene, um diese Veränderungen herbeizuführen, besteht darin, dass ich die befruchteten Seeigeleier etwa 10-20 Minuten nach der Befruchtung in verdünntes Seewasser bringe. Fur das Arbaciaei wird durch Zusatz von etwa $100 \%$ destillirten Wassers die nöthige Verdünnung erreicht. In Folge der osmotischen Druckdifferenz zwischen Eiinhalt und Seewasser dringt Wasser in das $\mathrm{Ei}$, sein Volumen nimmt zu und die Membran platzt. Man kann fast bei jeder Kultur, wenn man genügend probirt, eine solche Verdünnung des Seewassers finden, bei der fast alle Membranen in wenigen Minuten bersten. Sobald die Membran geborsten ist, beginnt das Protoplasma an der Rissstelle auszufließen. Das 
ansfließende Protoplasma nimmt sofort Tropfenform an. Textfig. 3 giebt schematisch in drei auf einander folgenden Stadien die Form des Extraovats wieder. Auf Grund der Arbeiten von Quincke müssen wir aus der Kugelform des Extraovats schließen, dass dasselbe an seiner Oberfläche mit einer Schicht einer mit Wasser nicht mischbaren Flüssigkeit (Öl) überzogen ist.

Gelegentlich aber findet das Ausfließen unter Wirbelbildung statt (Textfig. 4). Man sieht in diesem Fall, wie auch gelegentlich bei

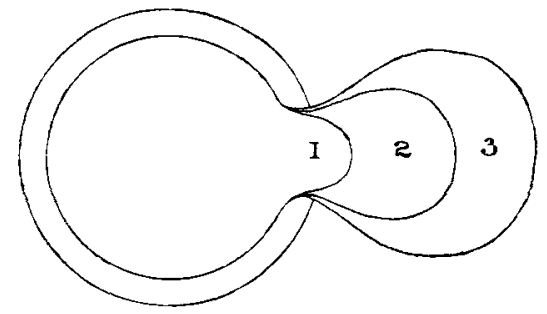

Fig. 3.

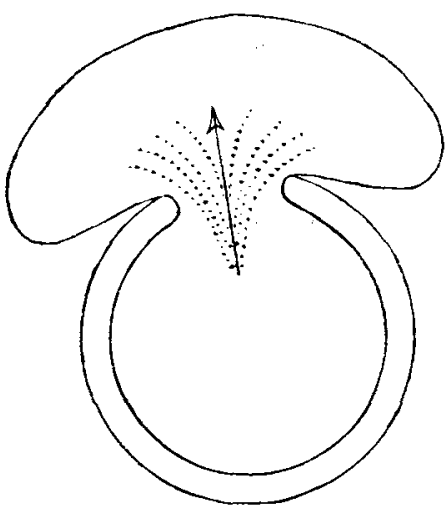

Fig. 4.

langsamem Ausfließen, im Centrum der Rissstelle ein rasches Strömen von Flissigkeitsmassen ans dem Ei in das Extraovat.

2. Wir können aber auch so verfahren, dass wir das Ei sich erst in normalem Seewasser furchen lassen und es dann in ver-

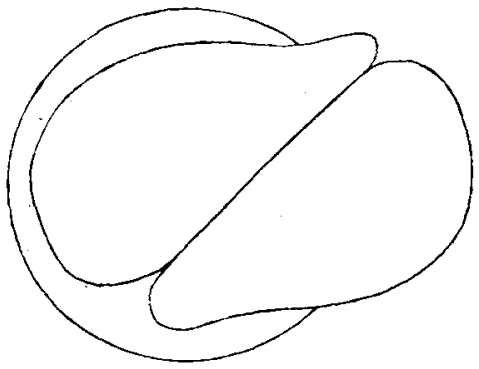

Fig. 5 .

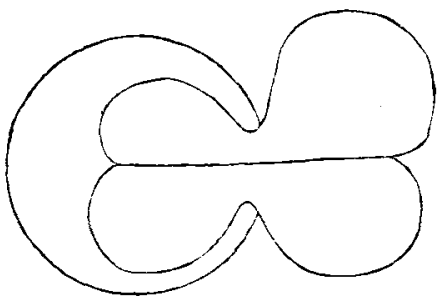

Fig. 6 .

schiedenen Furchungsstadien in verdünntes Seewasser bringen. Alsdann sehen wir das Extraovat sich ebenso bilden, als wenn das Ei nngefurcht wäre. Der einzige Untersehied ist der, dass das 
Beitr. z. Entwicklgsmech. d. ans einem Ei entstehend. Doppelbildungen. 459

Extraovat ans mehreren Zellen besteht. Textfig. 5 a. 6 stellen im Zweizellenstadium geplatzte Eier dar, Textfig. 7 ein im Vierzellenstadium und Textfig. 8 ein etwa im 32-Zellenstadium geborstenes Ei. DerUmstand, dass das Extraovat sich in all diesen Fällen ebenso verhält wie beim ungefurchten Ei, verliert alles Auffällige, wenn wir uns

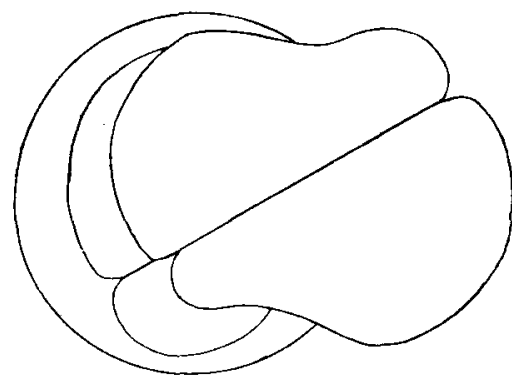

Fig. 7.

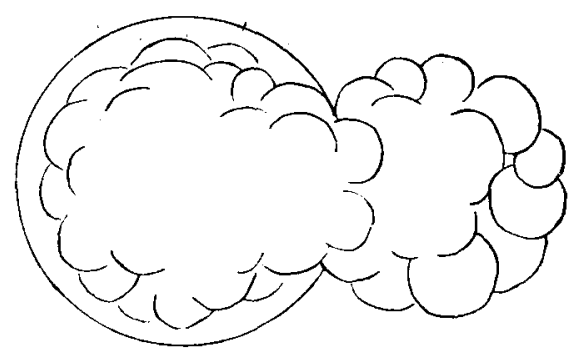

Fig. 8.

erinnern, dass nach Quincke und BÜTschli das Protoplasma Schaumstruktur besitzt und dass jede Furchungskngel eben nur ein Aggregat einer großen Zahl kleiner Schaumblasen ist. Das spätere Schicksal dieser Eier ist dasselbe wie das der vor der Furchung deformirten, mit denen wir uns im Folgenden weiter beschäftigen wollen.

3. So lange die Eier in hinreichend verdünntem Seewasser bleiben, tritt keine Furchung ein. Das Protoplasma befindet sich in $\times$ Wasserstarre. Eine solche Verdünnung des Seewassers, die nicht vollständige Wasserstarre herbeiführt, bewirkt wenigstens Verzögerung der Furchungsvorgänge. Ich fand es besser, die Eier länger als eine halbe Stunde im verdünnten Seewasser zu lassen, bevor ich sie in normales Seewasser zurickbrachte. Brachte ich sie vor dieser Zeit zurück, so schnürte sich das Extraovat leicht ab. Gewöhnlich blieben die Eier 2 Stunden in verdünntem Seewasser. In normales Seewasser zuruickgebracht, verliert die Doppelkugel Wasser und wird entsprechend kleiner. Die Furchung tritt gewöhnlich erst nach einer Stunde oder noch später ein, im Gegensatz zu den Eiern, deren Furchung durch den umgekehrten Vorgang, nämlich durch Wasserentziehung verhindert wird. Die Furchung ist bei den wasserstarr gewesenen Eiern meist eine Reihe auf einander folgender Zweitheilungen der einzelnen Zellen. Nach DrIEsch sollen Eier, die längere Zeit in verdinnntem Seewasser sich befanden, in mehr als zwei Zellen auf einmal zerfallen, wenn sie in normales 
Seewasser zurikekkommen. Das habe ich gelegentlich, aber doch recht selten beobachtet.

4. Die erste Furchungsebene, welche die Doppelkugel in zwei Zellen theilt, hat, wie ich schon frtiher angab, eine bestimmte Orientirung: sie steht im Allgemeinen auf dem gemeinsamen Durchmesser beider Kugeln senkrecht (Textfig. 9 u. 10). Das entspricht der Angabe von Roux und 0 . HerTwrg, dass die Kernspindel sich mit ihrer Längsachse in den größten Durchmesser des Protoplasmakörpers

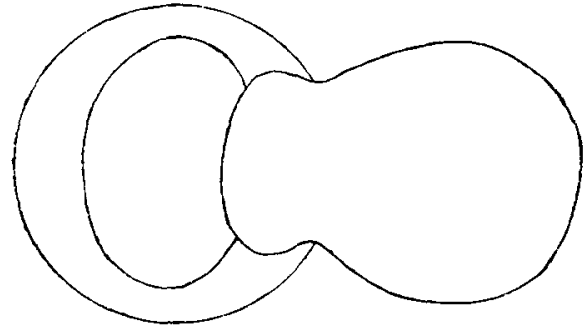

Fig. 9.

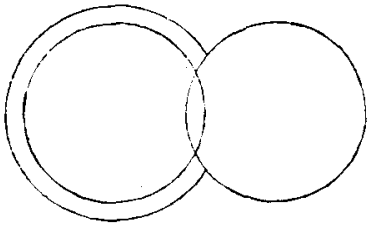

Fig. 10.

stellt. Ausnahmen kommen gelegentlich vor, lassen sich aber verstehen, wenn man beriucksichtigt, dass die Eier vorher in Wasserstarre sich befanden. Ob die erste Furchungsebene im Ei oder im Extraovat liegt, hängt davon ab, in welcher der beiden Kugeln der Kern sich befindet.

5. Von nun an verläuft die Furchung regelmäßig (Textfig. 11, 12 und 13), bis das Ganze in so kleine Zellen zerfallen ist, dass die

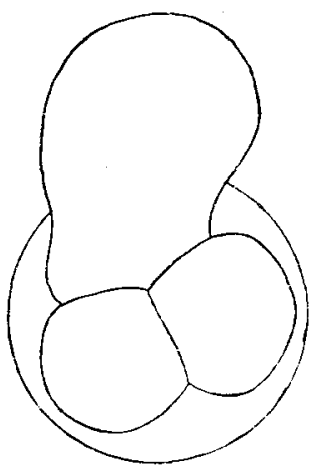

Fig. 11.

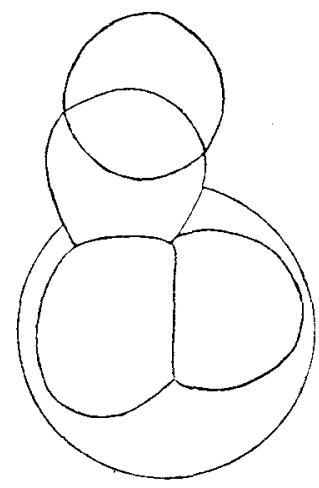

Fig. 12 .

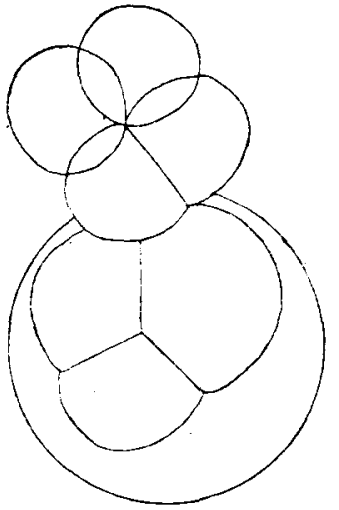

Fig. 13.

Bildung einer Hohlkugel (Blastula) stattfindet. Müssen sich die beiden Morulae zu einem oder zu zwei Blastulae entwickeln? Über den Mechanismus der Blastulabildung ist sehr wenig bekannt. Ich 
Beitr. z. Entwicklgsmech. d. aus einem Ei entstehend. Doppelbildungen. 461

bin geneigt denen beizustimmen, die folgende zwei Umstände als wesentlich hervorheben: 1) Die Adhäsion zwischen benachbarten Zellen der Oberfläche nimmt zu and 2) die Zellen secerniren Flüssigkeit in der Richtung von der Oberfläche der Morula gegen das Centrum derselben. Der letztere Umstand involvirt die Annahme, dass eine Polarität und Orientirung der Zellen besteht, da sonst nicht zu verstehen wäre, warum die Sekretion von Flìssigkeit nur in einer Richtung erfolge 1). Die Sekretion muss anch unter relativ hohem Druck stattfinden. Wenn wir uns nach der Reizursache umsehen, die eine solche Orientirung der Zellen herbeifuihren könnte, so könnten wir an das umgebende Seewasser denken. Sehen wir doch, dass nicht nur jede Blastula, sondern jeder kleine unregelmäßige Zellhaufen, der vom $\mathrm{Ei}$ abgetrennt wird, an seiner vom Seewasser berührten Seite Wimpern bildet. Da ferner die Eier vor der Blastulabildung specifisch schwerer sind als das Seewasser und rasch darin zu Boden sinken, während sie nach der Blastulabildung im Seewasser schwimmen, so ist auch an die Möglichkeit zu denken, dass die in der Blastulahöhle enthaltene Flüssigkeit specifisch leichter ist, als das Seewasser, und chemisch davon verschieden ist, obgleich freilich auch die Thätigkeit der Cilien den Auftrieb begünstigen muss. Wie gestaltet sich nun dieser Vorgang der Blastulabildung bei einer Doppelmorula wie Textfig. 14? In der Mehrzahl der Fälle bildet jede der beiden Kugeln eine besondere Furchungshöhle, gerade als ob wir es mit zwei von verschiedenen Eiern herrührenden Morulae

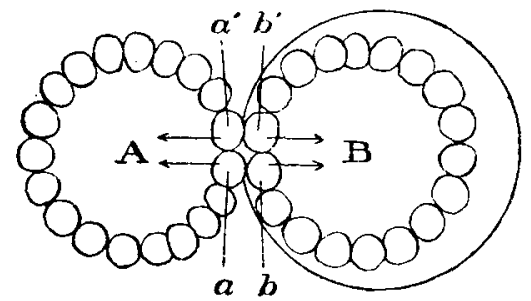

Fig. 14.

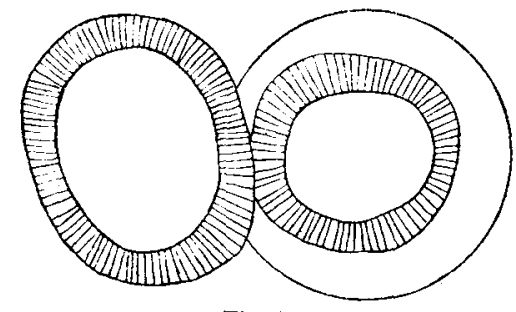

Fig. 15.

zu thun hätten. Eine Schwierigkeit für das Verständnis des Vorganges bereitet nur das Verhalten derjenigen Zellen $a a^{\prime}$ und $b b^{\prime}$ (Textfig. 14), die an der gemeinsamen Grenze der beiden Morulae $A$ und

1) Dieser Aufsatz war schon im Manuskript fertig als mir DRIEsch's nenes Buch "Analytische Theorie der organischen Entwickelung " durch die Freundlichkeit des Verfassers zuging; in dem ebenfalls eine Ordnung der Zellen der Blastula angenommen wird. 
$B$ liegen. Da diese Zellen an der dem nächsten Morulacentrum abgewendeten Seite nicht von Seewasser umspïlt sind, so ist kein Grund vorhanden, warum sie mehr gegen das Centrum der Morula $A$ als gegen das von $B$ secerniren sollten. Allein auch die Natur der in das Centrum der Morulae secernirten Fltissigkeit könnte einen orientirenden Einfluss auf die Zellen oder deren Inhalt haben. Geben wir das $z u$, so folgt, dass die Zellen $a a^{\prime}$ sich so orientiren mitssen, als ob sie zum System der Hohlkugel $A$ gehörten, während die Zellen $b b^{\prime}$ sich so orientiren müssen, als ob sie zum System der Hohlkugel $B$ gehören. Wir haben uns also vorzustellen, dass zunächst die Zellen $a a^{\prime} b b^{\prime}$ der Bertihrangsstelle beider Kugeln unthätig bleiben, während die tibrigen durch das Seewasser orientirten Zellen secerniren. Das Sekret der Kugel $A$ wirkt richtend auf die Zellen $a a^{\prime}$ das von $B$ anf $b b^{\prime}$ derart, dass die physiologische Innenseite der Zellen gegen das nächste Kugelcentrum gerichtet wird. Aus solchen Eiern entstehen zwei sich berïhrende Blastulae (Textfig. 15).

6. Es bleibt nun tibrig die kleinere Zahl der anderen möglichen Fälle zu betrachten. Der zweithäufigste Fall ist der, dass beide Morulae zusammen nur eine Furchungshöhle und folglich eine Blastula bilden. Um bei unserer Hypothese zu bleiben, müssen wir annehmen, dass die Zellen $a a^{\prime}$ und $b b^{\prime}$ ihren Inhalt in anderer Weise orientiren, als im vorigen Falle bei der Bildung von Zwillingen. In diesem Falle orientiren sich die Zellen $a$ und $b$ in gleichem Sinne, ebenso die Zellen $a^{\prime}$ und $b^{\prime}$. Alle richten ihre physiologische

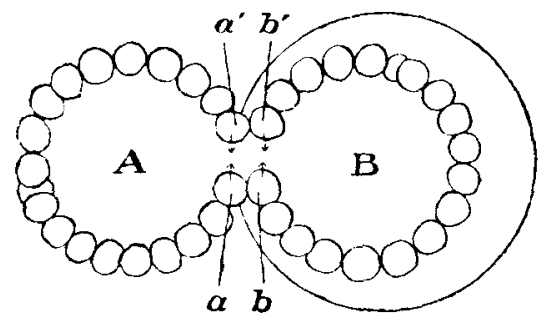

Fig. 16.

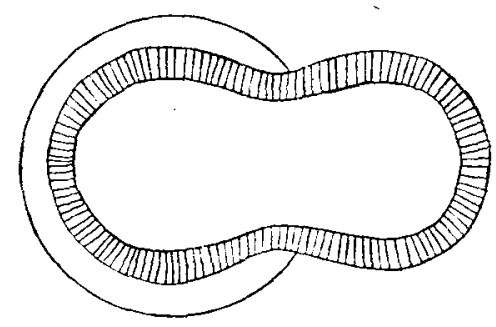

Fig. 17.

Außenseite gegen das Seewasser, die Innenseite gegen den gemeinsamen Durchmesser der beiden Morulae (Textfig. 16). Thatsächlich erhalten wir in einer Reihe von Fällen aus der Doppelkugel Blastulae von der Form der Textfig. 17. Die äußere Form der Doppelkugel ist gewahrt, aber es existirt nur eine Furchungshöhle. Entsprechend entsteht anch nur ein Embryo, der aber, wie wir bald sehen werden, Ansätze zur Doppelbildung enthalten kann. Blastulae 
von der Form der Textfig. 16 hat schon Driesch gelegentlich bei Eiern beobachtet, die er im Zwei- oder Vierzellenstadium geschuttelt hatte. Unsere Methode gestattet, diese Blastulae nach Belieben in jedem Stadium hervorzubringen.

7. Ein dritter seltenerer Fall ist endlich der, dass wir mehr als zwei Blastulae aus einer Doppelkugel erhalten. Das geht meist so zu, dass im Extraovat vor der Blastulabildung Verschiebungen von Zellen oder Zellgruppen stattfinden, so dass wir zur Zeit der Blastulabildung nicht eine einzige Doppelkugel vor uns haben, sondern drei oder mehr Morulae. Für die Bildung von Blastulae gilt dann fü je zwei dieser Kugeln dasselbe Raisonnement, das wir vorher angewendet haben. Einen instruktiven Fall dieser Art habe ich in meinem Aufsatz *Über die Grenzen der Theilbarkeit der Eisubstanz * mitgetheilt. Die Bewegungen der Zellen sind von großer Bedeutung für die Entstehung von Doppelbildungen und für die Organbildung überhaupt, und es ist daher der Mühe werth, die Frage aufzuwerfen, welche Kräfte diese Bewegungen bestimmen. Der einfachste Fall einer solchen Bewegung der Zellen ist vielleicht der folgende. Wenn man normale Eier im Zweizellenstadium in hinreichend verduinntes Seewasser bringt, so dass die Membran platzt, so fließt oft nur ein Theil des Protoplasmas aus einer der beiden Kugeln aus (Textfig. 18). Diese Form ('Textfig. 18) kann sich wegen der Oberflächenspannung des Extraovats nicht erhalten. Das letztere nimmt Kugelform an, und das Ganze ordnet sich symmetrisch um den durch das Centrum der Ausflussöffnung gelegten Durchmesser des ursprtinglichen Eies Textfig. 19). Eine derartige auf Oberflächen-

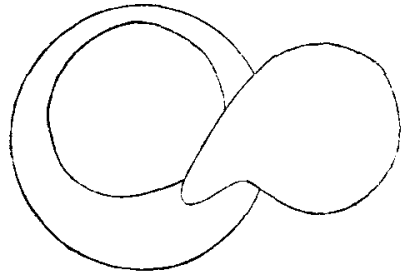

Fig. 18.

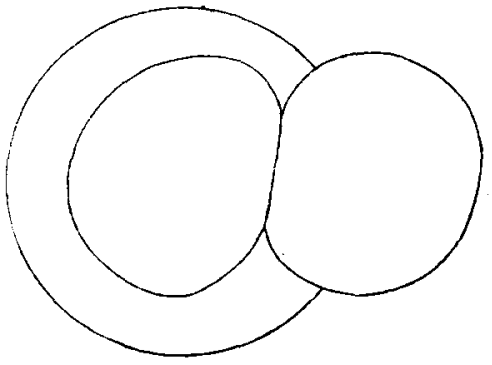

Fig. 19.

spannung beruhende Umordnung der Massen findet fast ausnahmslos statt, sobald ein solches Ei in normales Seewasser zurückgebracht wird, ein Beweis, dass hier der Eiinhalt und seine Oberfläche flusssíg ist (obwohl feste Partikel eingelagert sein können). Auch im ver- 
dünnten Seewasser können derartige Umlagerungen vor sich gehen, aber so viel ich sehen konnte, immer nur unmittelbar nach dem Platzen des Eies, bevor die "Wasserstarre * eintritt.

Es ist bekannt, das jedes Mal, "sobald die Zellen zur Theilung sich anschicken, ihre Oberfläche sich mehr der Kugelform nähert. Was auch immer die Ursache dieser Änderungen sein möge, es ist klar, dass, wie in dem eben erwähnten Falle, diese Änderungen in der Form der Oberfläche zu Verschiebungen einzelner Zellen oder Zellgruppen führen kann, namentlich im Extraovate, wo der Beweglichkeit der Zellen keine Grenzen durch die Membran gesteckt sind. So ist es nicht sonderbar, dass aus dem Extraovat sich leichter mehr als eine Morula und Blastula bilden kann, als ceteris paribus aus dem in der Eihaut gebliebenen Plasma.

\section{Die weitere Entwickelung der deformirten Eier.}

1. Die [weitere Entwickelung der Doppelkugeln, welche nur eine einzige Furchungshöhle bilden, hängt von dem Verhalten der Eihaut_ab: Fällt dieselbe im Laufe der ersten 24 Stunden ab, oder verliert sie ihre Spannung, so wird der intrablastuläre Druck ausreichen, die Kugelform der Blastula mehr oder weniger vollständig wieder herzustellen, wie DrIEsch schon beobachtet hat. Gastrula und Pluteus sind alsdann normal. In manchen Kulturen aber blieb die Membran mit starker Spannung erhalten. Fig. 1, 2 und 3 auf Taf. XVIII zeigen die beginnende Skeletbildung in diesen deformirten Embryonen. Die Plutei, die sich unter diesen Umständen entwickelten, hatten nur einen Darmkanal, aber die Skeletbildung war häufig abnorm und zeigte alle Übergänge von einfacher Verlagerung eines Armes (Fig. 4 u. 5 auf Taf. XVIII) bis zur beginnenden resp. vollendeten Verdoppelung des Skelettes (Fig. 6, 7, 8 u. 9 auf Taf. XVIII. Die Buchstaben $a$ und $b$ bezeichnen in diesen Figuren die durch die Membran hervorgebrachte Einschntirung an der Grenze von Extraovat und Eiinhalt. Fig. 6 (Taf.XVIII zeigt besonders deutlich an der Einschniirungsstelle einen Ansatz zur doppelten Skeletbildung. Diese Figur wie Fig. 8 (Taf. XVIII) sind von Embryonen angefertigt, welche derselben Kultur angehörten wie die Fig. 1, 2 und 3 (Taf. XVIII). Andere Abnormitäten des Skelettes wird der mit dem Gegenstand vertraute Leser leicht herausfinden, z. B. in Fig. 6 (Taf. XVIII) die Einsehiebung eines Zwischenstücks $c$ und. $d$ zwischen die beiden Arme.

2. Bilden sich zwei oder mehr Blastulae, so entstehen auch zwei oder mehr Plutei, falls die Stücke, aus denen sie sich entwickeln, nicht zu wenig Masse haben. Die aus demselben Ei entstehenden 
Beitr. z. Entwicklgsmech. d. aus einem Ei entstehend. Doppelbildungen. 465

Blastulae sind Anfangs mit einander verklebt, sie werden aber meist, sobald die Blastulae zu schwärmen beginnen, mechanisch von einander getrennt. Im letzteren Falle erhalten wir normale wenn auch zwerghafte Plutei. Findet die Trennung aber nicht statt, so erhalten wir zusammengewachsene Doppel- oder Mehrfachembryonen. Die Orientirung der zusammengewachsenen Doppelembryonen gegen einander lässt keine bestimmten Regeln erkennen. Ebenso kann auch die Ausdehnung der Verwachsung verschieden sein. In Bezug auf die Orientirung können wir symmetrische und unsymmetrische Zwillinge unterscheiden. Die Fig. 10, 11, 12 auf Taf. XVIII und 13, 1415 auf Taf. XIX sind mehr oder weniger vollkommen symmetrische Zwillingsbildungen, in Fig. 16 und 17 (Taf. XIX) haben wir es mit unsymmetrisehen Verwachsungen zu thun. Die symmetrischen Bildungen überwiegen nach meinen Erfahrungen. Fig. 18, 19 und möglicherweise auch Fig. 20 (Taf. XIX) sind Drillingsplutei. Die letzteren sind sehr selten. Vierlinge beobachtete ich niemals im Pluteusstadium, wie ich glaube desshalb, weil die Entwickelungsfähigkeit um so frïher ihr Ende erreicht, je kleiner der Bruchtheil des Eies ist. Wenn desshalb auch gelegentlich richtige Vierlinge im Blastulastadium zur Beobachtung gelangen, so ist doch wenig wahrscheinlich, dass alle Blastulae das Pluteusstadium erreichen. $\mathrm{Zu}-$ dem ist es sehr wenig wahrscheinlich, dass nicht eine oder mehrere Blastulae bei der Bewegung abgetrennt werden sollten.

Wenn Doppelplutei aus zwei Blastulae entstehen, hat in der Regel jeder einen besonderen Darmkanal, wie es in Fig. 10-19 auf Taf. XVIII u. XIX der Fall ist.

\section{IV. Über das Verhalten von Eiern, die vor der Befruchtung und im Blastulastadium in verdünntes Seewasser gebracht werden.}

1. Unbefruchtete reife Eier nehmen in verdünntem Seewasser an Volumen zu, aber da sie keine Membran besitzen, so findet auch keine Extraovatbildung statt. Sie nehmen aber, wie ich friher angab, weniger Wasser auf als befruchtete Eier. Das Spermatozoon löst also im Ei Vorgänge aus, die zu einer Zunahme der Zahl der gelösten Molektule fuhrt. Diese durch den Eintritt des Spermatozoons herbeigeftibrten Änderungen im $\mathrm{Ei}$ sind also in Bezug auf den osmotischen Druck ähnlich den Änderungen im thätigen Muskel ${ }^{1}$. Ich gab mir viele Mühe festzustellen, ob eine Wärme-

1) Vgl. meine Notiz „Über die Entstehung der Aktivitätshypertrophie der Muskelns. PflüGer's Archiv. Bd. 56. 
bildung bei der Befruchtung stattfinde, bis jetzt aber ohne positiven Erfolg. Ich bin aber mit der Fortfuhrung dieser und weiterer Versuche über die Befruchtung beschäftigt und will daher auf diesen Gegenstand hier nicht weiter eingehen. Es scheint mir auch, dass mit den Zelltheilungsvorgängen periodische Sekretionsvorgänge verlaufen.

2. Bringt man Eier, die eben ins Blastulastadium getreten sind, in verdünntes Seewasser, so platzt die Membran und ein Prolaps der Blastula findet statt (Textfigur 17). Dagegen habe ich mich nicht ubberzeugen können, dass die Wand der Blastula selbst jemals platzte. Brachte man das Ei in normales Seewasser zurück, so nahm die Blastula wieder Kugelform an. Aus diesen Blastulae entwickelten sich immer nur einfache Embryonen, da eben nur eine Blastulahöhle vorhanden war.

Die Blastulae bleiben im verdtunnten Seewasser viel länger am Leben und entwicklungsfähig, als in den ersten Furchungsstadien befindliche Eier. Auch gegen koncentrirteres Seewasser sind die Blastulae resistenter. Ähnliche Änderungen der Empfindlichkeit gegen Änderungen des Wassergehaltes in verschiedenen Entwicklungsstadien fand ich früher bei Fischeiern (PflüGer's Archiv. Bd. 54.

Eine Möglichkeit, die nicht nur für die hier erwähnte Prolapsbildung der Blastulae, sondern auch für das Ausfließen des Extraovats frisch befruchteter Eier in Betracht kommen könnte, möchte ich wenigstens erwähnen. Es wäre denkbar, dass das Ausfließen des Protoplasmas an der Rissstelle der geplatzten Membran zum Theil auch durch physikalische Ausbreitungserscheinungen an der Grenze von Eiinhalt und Seewasser mitbestimmt sei.

\section{v. Zur Mechanik der Spaltungsvorgänge des Keimes, welche zu Doppelbildungen führen.}

1. Die vorher mitgetheilten Versuche zeigen insbesondere, wie eine selbst partielle Spaltung des Keimes zu partiellen und zusammengewachsenen Doppelbildungen fuihren kanu. Sie zeigen ferner, dass auch getrennte $Z$ willinge, sogar in der Mehrzahl der Fälle, entstehen. Es ist klar, dass die osmotische Druckdifferenz, die zur Umformung des kngelförmigen Eies in eine Doppelkugel nöthig ist, auch zwischen Eiinhalt und den Flüssigkeiten des Uterus, in erster Linie dem Blute, vorhanden sein kann, wenn nämlich das Ei einen abnorm hohen osmotischen Druck besitzt, also insoferu " pathologisch * ist. In der That habe ich gefunden, dass auch ein allerdings 
kleiner Procentsatz von Seeigeleiern jin normalem Seewasser platzt und zu Doppelbildungen Veranlassung geben kann. Auf der anderen Seite muss ich aber betonen, dass bei manchen Eiern das Sprengen der Membran durch Wasseraufnahme nicht gelingt, z. B. bei Annelideneiern (nach Versuchen von Mr. MEAD und mir selbst).

2. Wir wollen uns nunmehr der Frage zuwenden, welche Vorgänge bestimmen eine Spaltung des Keimes bei unversehrter Eihaut? Außer den in der Einleitung angegebenen Beobachtungen kann ich hinzufügen, dass ich die Entwicklung von Doppelbildungen nicht selten beobachtete bei Seeigeleiern, deren Furchung gleich nach der Befruchtung in hinreichend verdinntemSeewasser fur 1-2 Stunden gehemmt worden war, deren Membran aber nicht platzte. In normalem Seewasser bildeten sich später in solchen Eiern zuweilen Doppelembryonen wie in Textfigur 20. Allein auch wenn befruchtete ungefurchte Eier erst in Seewasser von höherer Koncentration gebracht wurden, das die Furchung unterdrickte, so trat auch gelegentlich eine Bildungron Doppelembryonen ein, wenn die Eier sich nachher in normalem Seewasser entwickelten. Die mannig-

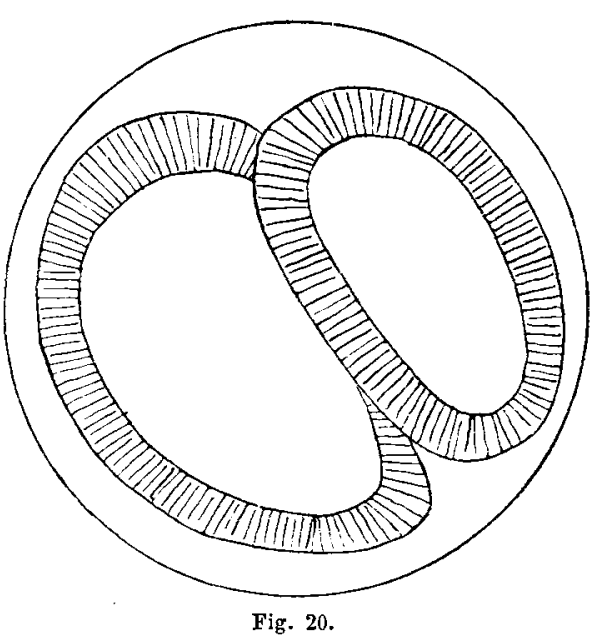

faltigsten äußeren Eingriffe, die am ungefurchten oder im Zweizellenstadium befindlichen Ei stattfinden, können also zu einer Zwillingsbildung führen. Im Allgemeinen kann man finden, dass in einem solchen Falle die ersten zwei Zellen von einander getrennt werden, oder dass in späteren Furchungsstadien die Zellen in zwei Gruppen gespalten werden. Wie kommt nun diese Trennung zu Stande? Um auf diese Frage eine Antwort zu erhalten, miissen wir den Vorgang der Furchung in solchen Eiern etwas genauer studiren.

Meine Beobachtungen über die Furchung von Eiern unter abnormen Bedingungen erinnerten mich an die Erscheinungen, die man wahrnimmt, wenn man einen Tropfen Sodalösung auf öl bringt, das freie Fettsäure enthält. Es kommt bekanntlich alsdann 
zu mehr oder weniger stürmischen amöboiden Bewegungen und unter gewissen Bedingungen zur Emulsionsbildung. Die Erklärung dieses Vorgangs ist nach Quincke die folgende: „ Bei der Bertihrung von öl und Sodalösung bildet sich feste Seife durch Einwirkung der im öl enthaltenen freien Fettsäure. Allmählich löst. sich ein Theil dieser Seife in der angrenzenden wässrigen Flüssigkeit. Die flüssige Seifenlösung breitet sich, sobald sie mit dem Öl in Berührung kommt, plötzlich an der Grenze von öl und wässriger Flüssigkeit aus und reißt die ungelösten Seifentheilchen und anhängenden Ölmassen mit fort. Dabei werden Ölfäden abgerissen und in die wässrige Flüssigkeit hineingezogen. Diese Ölfäden haben das Bestreben, möglichst kleine Oberfläche anzunehmen und zerfallen in kleinere oder größere Tropfen. . . . Diese periodischen Ausbreitungen von Seifenlösung an der Grenze von Öl und wässriger Fluissigkeit erfolgen nicht gleichzeitig an allen Punkten der Öloberfläche. Sie sind mit den oben beschriebenen Wirbelbewegungen im Inneren beider Flüssigkeiten verknüpft, welche die wässrige Flüssigkeit und besonders das zähflüssige Öl nach dem Ausbreitungscentrum hinziehen. Das giebt die amöbenartigen Bewegungen am Rande der Ölmasse. . . . Bei einer mittleren Größe der Zähigkeit des Öles und der Stärke der Ausbreitung an der Grenze von öl und wässriger Flüssigkeit mutssen die Wirbelbewegungen und die Anzahl der losgerissenen ölkugeln besonders groß sein 1). " Quincke weist dann ferner darauf hin, dass, wenn die Seife zu schnell sich bildet, oder wenn sich zu wenig bildet, oder wenn sie zu schnell aufgelöst wird, die amöbenartigen Bewegungen ausbleiben müssen und dass ferner die Zähigkeit des Öls eine Rolle bei dem Ablauf der Erscheinungen spielt. Da nun diese Umstände nicht nur von den inneren Bedingungen des Systems allein, sondern auch von äußeren Bedingungen, z. B. der Temperatur abhängen, so lässt sich einsehen, dass diese amöboiden Bewegungen durch die äußeren Bedingungen verhindert, verringert oder verstärkt werden können.

Nach Quincke sind nun die Bedingungen für das Stattfinden soleher Ausbreitungserscheinungen beim lebenden Protoplasma vorhanden und die diese Ausbreitungsvorgänge begleitenden Wirbelbewegungen im $\mathrm{Ei}$ sind es meines Erachtens, welche nicht nur nach vollendeter Kerntheilung die normale Zellfurchung herbeiführen,

1) G. QUincke, fÜber periodische Ausbreitung an Fliissigkeitsoberflächen und dadurch hervorgerufene Bewegungserscheinungen. Wiedemans's Annalen. Bd. 35. 1888. 
sondern die auch, wenn sie stürmischer verlaufen, zur Spaltung des Keimes und damit zur Zwillingsbildung führen müssen. Nur mit dem letzteren Fall wollen wir uns hier beschäftigen.

3. Im Seeigelei befindet sich zwischen Membran und Protoplasma eine wässrige Flussigkeit, die von außen durch die Eihaut diffundirt. Wie die Beobachtungen am Extraovat sowie die Kugelform der Furchungszellen zeigen, muss die Oberfläche des Protoplasmas von einer mit Wasser nicht mischbaren Flisssigkeit umgeben sein und das kann nach Quincke nur eine Ölschicht sein, die jedoch außerordentlich dïnn sein kann. Es ist ferner von Quincke nachgewiesen, dass das Eiweiß zur Herbeiführung von Ausbreitungserscheinungen an der Grenze von Öl und Wasser in derselben Weise geeignet ist wie Sodalösungen. Nun hat allerdings noch Niemand das Stattfinden von Ausbreitungserscheinungen an der Oberfläche der Furchungskugeln bei ihrer Theilung direkt gesehen. Dagegen ist es eine allgemein bekannte Thatsache, dass Änderungen in der Form der Oberfläche der Zellen während der Furchungsvorgänge stattfinden. Dieselben nähern sich mehr der Kugelform und trennen sich mehr von den benachbarten Zellen, während sie nach abgelaufener Furchang sich wieder auf einer größeren Oberfläche berïhren. Ich stelle mir nun vor, dass an der Oberfläche des Eies, möglicherweise in demjenigen Meridian oder Kreise, dessen Ebene die beiden Strahlensysteme der Centrosome von einander trennt, sobald die Kerntheilung physikalisch abgelaufen ist, Ausbreitungserscheinungen stattfinden. Dieselben führen zur Bildung von in Bezug auf diese Ebene symmetrischen Wirbelbewegungen. Während diese Wirbelbewegungen nun im s normalen * $\mathrm{Ei}$ und unter snormalen * Bedingungen ruhig verlaufen und nur zur Zerreißung der Oberfläche, d. h. zur Furchung, führen, kann es unter gewissen abnormen äußeren Bedingungen oder bei etwas abnormer Beschaffenheit des Eies zu stürmischeren Ausbreitungserscheinungen und Wirbelbewegungen kommen, die dazu führen, dass eine Schicht der wässrigen Flussigkeit sich zwischen die beiden Furchungskugeln legt und dieselben trennt. In Folge dieser Trennung mitssen sich beide Zellen unabhängig von einander zu vollkommenen Embryonen entwickeln.

4. Wenn diese Theorie richtig ist, welche die Bildung von Zwillingen bei intakter Eihaut auf stürmische Wirbelbewegungen in den Furchungszellen und der sie umgebenden wässrigen Flüssigkeit zurückführt, so müssen wir auch erwarten, hierbei Erscheinnngen 
zu beobachten, welche derartige energische Ausbreitungsvorgänge begleiten, nämlich das Abreißen von Tropfen and das Auftreten amöboider Bewegungen. Derartige Beobachtungen machte ich bei Gelegenheit der Versuche tiber Kerntheilung ohne Zelltheilung, welche ich vor 2 Jahren veröffentlichte ${ }^{1)}$. Ich hatte Eier nach der Befruchtung in Seewasser gebracht, dessen Koncentration durch Zusatz von Kochsalz erhöht war. In dieser Lösung fand bei passender Koncentration eine Kerntheilung, aber keine Zelltheilnng statt. Brachte ich die Eier nun, nachdem mebrere Kerne sich gebildet hatten, in normales Seewasser zurtick, so fanden stirmische, wogende Protoplasmabewegungen statt, die zur Zelltheilung führten, wobei es aber häufig vorkam, dass Protoplasmamassen, die eben durch eine Furche getrennt waren, wieder zusammenflossen. Auch hierbei kam es nicht selten zur Entstehung von zwei Blastulae in einem Ei. Dasselbe beobachtete dieses Jahr Mr. W. W. Norman, der diese Versuche in meinem Laboratorium wiederholte. Ebenso harmonirt damit die folgende Angabe von Driescr iber die Furchung von Seeigeleiern bei höherer Temperatur. "Die Zweitheilung der Versuchsobjekte bietet meist folgendes Bild. Es gewähren kurz gesagt die einander zugewandten Flächen beider Furchungszellen den Eindruck, als wären sie aus einander gerissen worden, sie berühren sich nicht, lassen vielmehr einen dentlichen Zwischenraum frei, erscheinen, von der Kante gesehen, unregelmäßig zackig und oft ist selbst von einer der Zellen ein Stïck Protoplasma in Tropfenform abgerissen, das jedoch wieder mit ihr verschmelzen kann. « DrIEsCH hat nun festgestellt, dass derartige Eier Zwillingen den Ursprung geben. Mr. Norman hat auch diese Beobachtung bestätigen können.

In den Versuchen von Schultze werden offenbar Bewegungen des Zellinhaltes durch die Anderung der geotropischen Orientirung des Eies hervorgerufen. Da mir aber diese Erscheinungen einstweilen nicht aus eigener Anschauung bekannt sind, so will ich nicht weiter auf dieselben eingehen.

Allein atch bei normalen äußeren Bedingungen kann es zu Trennungen der beiden ersten Furehungszellen kommen. Die einzelnen Eier zeigen nämlich individuelle Verschiedenheiten. Es wird sich desshalb stets ein gewisser Procentsatz finden, bei dem die Viskosität des Protoplasmas oder die Verhältnisse der Bildung und

1) Experiments on Cleavage, Journal of Morphology Vol. VII. 
Beitr. z. Entwicklgsmech. d. aus einem Ei entstehend. Doppelbildungen. 471

Lösung der , Eiweißseifen a gerade der Art sind, dass die Ausbreitungsvorgänge mit besonderer Energie ablaufen. Das muss bei den im Uterus sich entwickelnden Eiern, aus denen mehr als ein Embryo hervorgeht, der Fall sein, da ja im Lterus die änßeren Bedingungen konstant sind.

Aach die Thatsache, dass so viele verschiedene Umstände im Stande sind, Doppelbildungen hervorzurufen, wird selbstverständlich mit der Annahme, dass starke Ausbreitungsvorgänge und Wirbelbildungen diesem Vorgange zu Grunde liegen. Es ist ja schon von Quncke gezeigt worden, dass sehr verschiedene Umstände die Energie der Ausbreitungserseheinungen verstärken können.

5. Wir dürfen aber nicht übersehen, dass auch noch andere Bedingungen hinzukommen können, welche eine Spaltung des Keimes begtinstigen. Dahin gehört vor Allem relativ geringes Volumen des Protoplasmas. Wenn die beiden ersten Furchungszellen den Raum in der Membran nicht ausfüllen, so können sie eher von einander getrennt werden. Das Volumen des Protoplasmas ändert sich aber mit seinem Wassergehalt und so werden Verringerungen des letzteren ein begünstigendes Moment bilden für die Trennung der beiden ersten Furchungskugeln durch eine sich zwischen dieselbe legende Schicht einer wässrigen Flüssigkeit. Ferner wäre es denkbar, dass die Adhäsion zwischen benachbarten Zellen durch abnorme Beschaffenheit der Oberfläche der Furchungskugeln verringert sein könnte. Es ist endlich auch kaum nöthig besonders hervorzuheben, dass bei solchen Organen, die durch Verwachsung zweier Hälften entstehen, das Ausbleiben der Verwachsung zur Verdoppelung führen könnte.

6. Ich brauche auch kaum noch zu betonen, dass nach dieser Theorie die Doppelbildungen bei intakter Eibaut eine Funktion derselben molekularen Vorgänge sind, welche auch die normale Furchung herbeiftihren, nämlich Ausbreitungsvorgänge und Wirbelbewegungen im Sinne von Qunvcke; und dass die Zwillingsbildung eben dann zu Stande kommt, wenn die die Furchung bestimmenden Wirbelbewegungen mit zu großer Energie verlaufen. Ist die Energie dieser Wirbelbewegungen zu klein, so tritt eine andere Erscheinung ein, die aber ebenfalls wohlbekannt ist, nämlich Kerntheilung ohne Zelltheilung. Wie sich diese Vorgänge im Einzelnen ableiten lassen, will ich in einer späteren Arbeit über die Mechanik der Zelltheilung auszuführen versuchen. 
7. In Bezng auf die Entstehung von Doppelbildungen fuihrt desshalb unsere Arbeit zu folgenden Ergebnissen:

1) Wenn die osmotische Druekdifferenz zwischen Eiinhalt und umgebender Flüssigkeit zu groß wird, sei es dass der osmotische Druck im Ei zu hoch oder in der umgebenden Flüssigkeit zu niedrig ist, kann das Volumen des Eies durch Wasseraufnahme zu groß werden und die Membran platzen. Bleibt der dabei austretende Theil des Protoplasmas mit dem im Ei befindlichen im Zusammenhang, so kann es zur Doppelbildung kommen.

2) Bei intakter Eihaut können ähnliche zur Doppelbildung führende Trennungen der ersten Furchungszellen dadurch hervorgerufen werden, dass stürmische Protoplasmabewegungen, sei es durch abnorme äußere Bedingungen, sei es durch abnorme Beschaffenheit des Eiinhaltes bei den ersten Furchungen im Ei ablaufen. Im Anschluss an Qunccke's Theorie der amöboiden Bewegungen können wir uns vorstellen, dass die Furchung des Protoplasmas durch Wirbelbewegungen herbeigeführt wird, die im Fall der Doppelbildung eine solche Stärke erreichen, dass die Furchungszellen durch eine Schicht wässriger Flüssigkeit von einander getrennt werden.

Bemerkung zu den Abbildungen:

Von den dieser Abhandlung beigefügten Abbildungen sind die Textfiguren 5, 6, 7, 8, 11, 12, 13, 15, 17, 18, 20 und alle Figuren der Tafeln XVIII u. XIX nach der Natur gezeichnet and zwar bis auf wenige Ausnahmen mit dem Zeichenprisma. Die übrigen Figuren sind mehr oder weniger schematisch. 
8
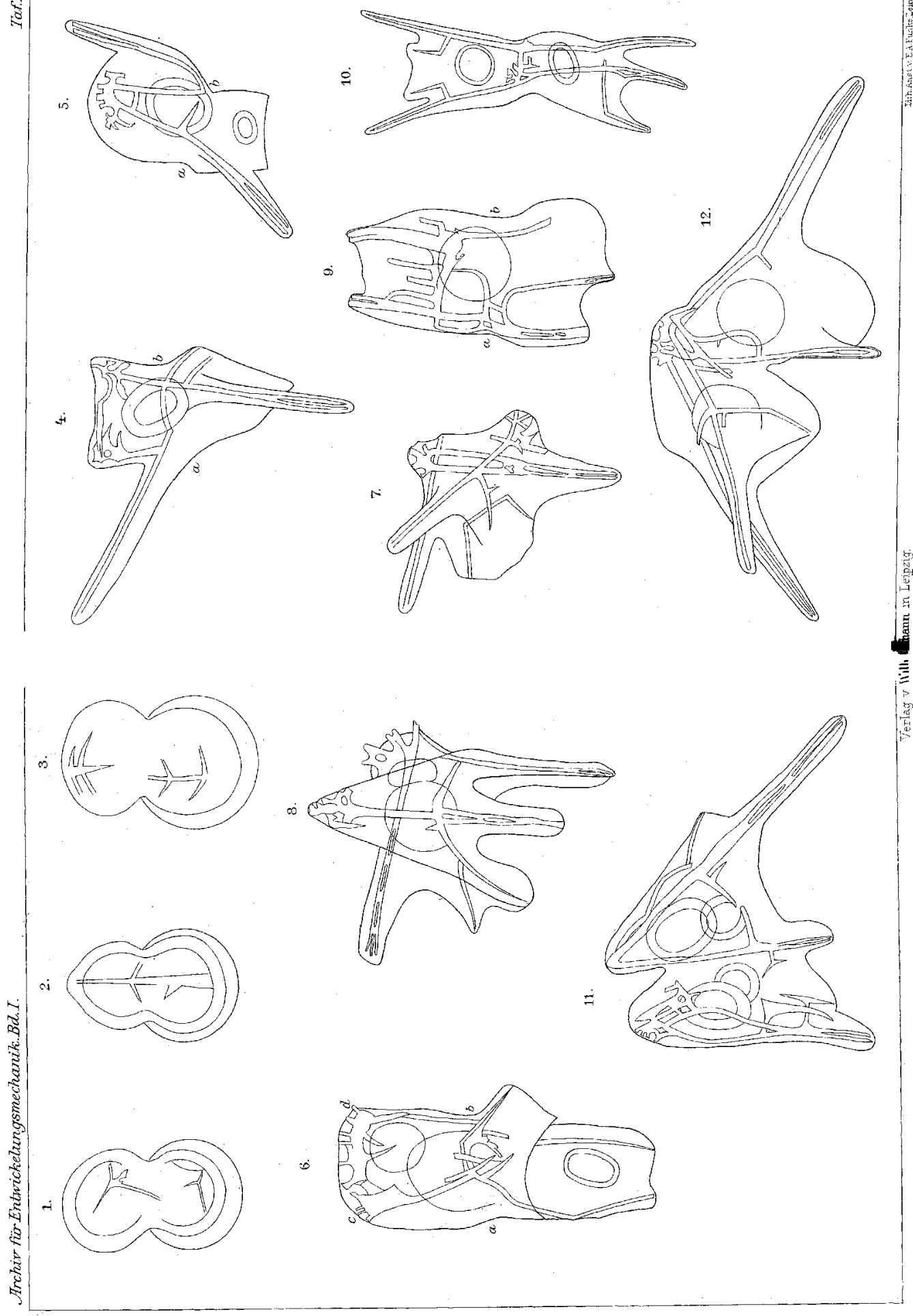
s
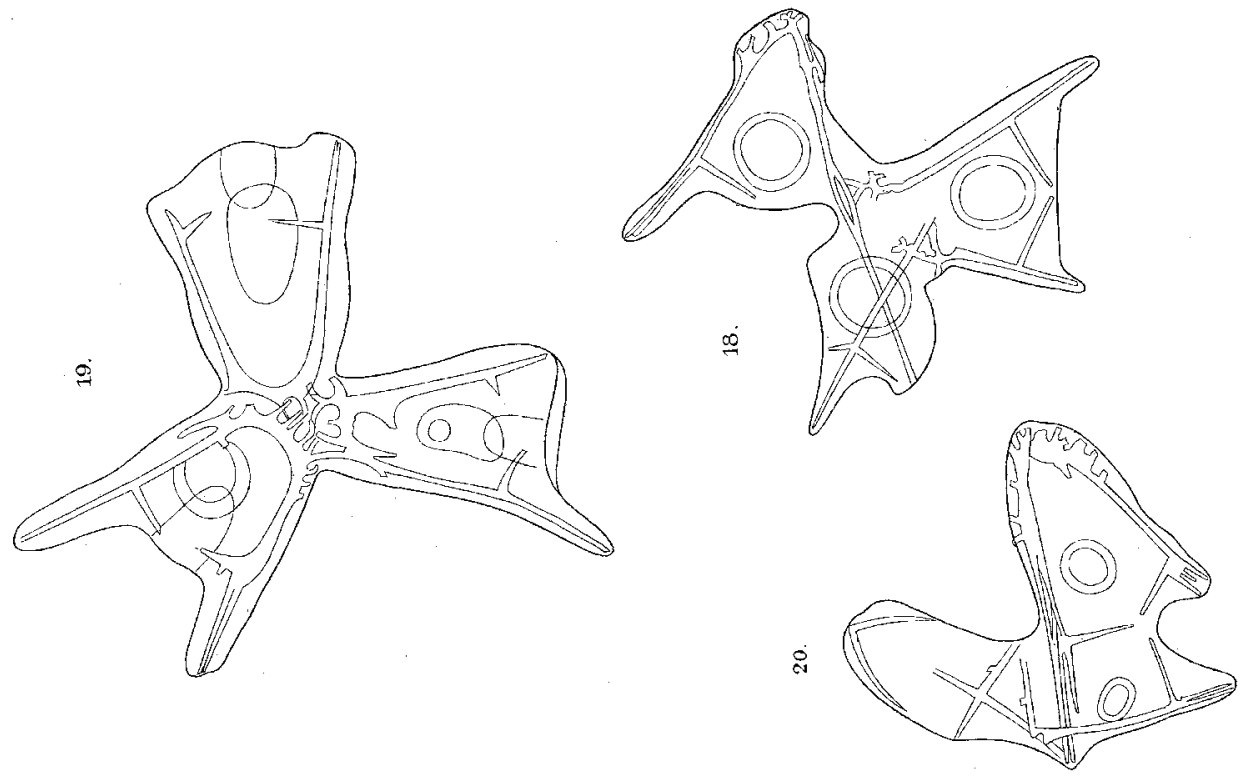

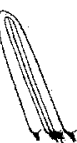
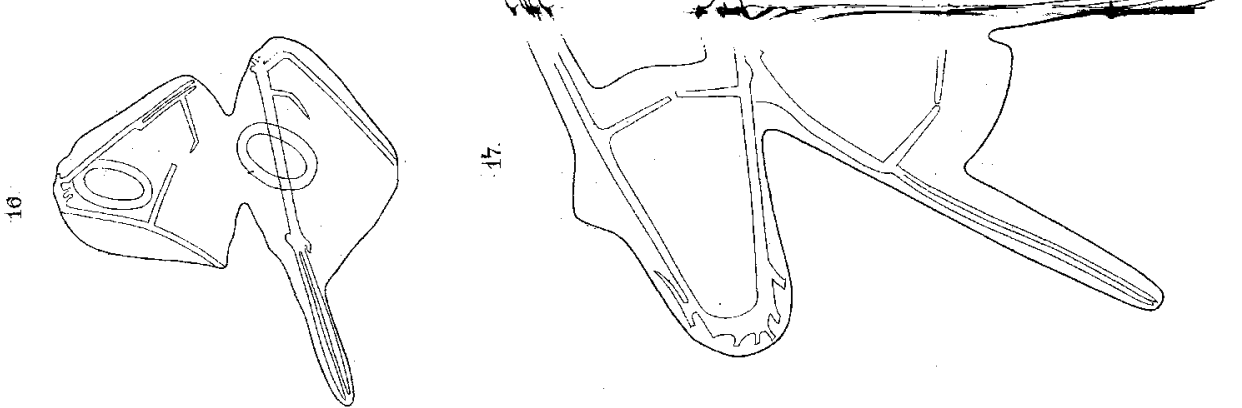

言
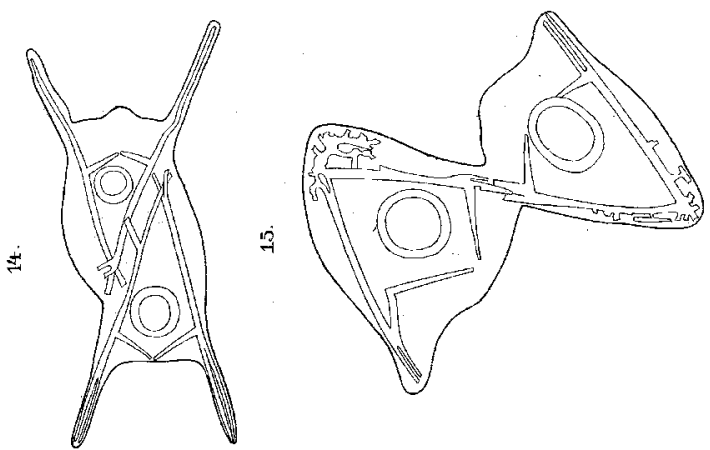